\title{
A PCA-based Face Recognition Method by Applying Fast Fourier Transform in Pre-processing
}

\author{
Dehai Zhang', Da Ding ${ }^{1}$, Jin $\mathrm{Li}^{1}$, Qing Liu ${ }^{1}$
}

\begin{abstract}
Principal Component Analysis (PCA) is a well-studied method in face recognition. Noticing that few researches focus on pre-processing of images, which will also improve the performance of feature extraction of PCA algorithm, we present an improved approach of PCA based face recognition algorithm using Fast Fourier Transform (FFT). In our method, FFT is presented as a method to combine amplitude spectrum of one image with phase spectrum of another image as a mixed image. PCA is applied to do feature extraction and a kernel SVM is harnessed as a classifier. To test and evaluate the performance of the proposed approach, a series of experiments are performed on Yale face database A. The experimental results show that our proposed method is encouraging.
\end{abstract}

Keywords: Face recognition; pre-processing; Fast Fourier Transform; PCA; SVM

\section{Introduction}

Face recognition is the most amazing of its theoretical and practical value for our daily life. Tasks like in airports, employee entries, criminal detection systems and identification based on face features will provide us with more accurate results [1]. However, as there are too many features in face or facial expression, most of these methods make trade-offs like hardware requirements, time to update image database, time for feature extraction, response time, etc [2]. So generally

\footnotetext{
1 Dehai Zhang $(\bowtie)$

School of Software, Yunnan University,Kunming, China e-mail: dhzhang@ynu.edu.cn

1 Da Ding

School of Software, Yunnan University,Kunming, China e-mail: dingda6@126.com

1 Jin Li

School of Software, Yunnan University,Kunming, China e-mail: lijin@ynu.edu.cn

1 Qing Liu

School of Software, Yunnan University,Kunming, China

e-mail: liuqing@ynu.edu.cn
} 
researchers would like to choose a special method that will reduce the amount of calculation and thus make the experiment much more efficient. Principal Component Analysis (PCA) is a well-studied method to extract feature sets by making sample projection from higher dimension to lower dimension [3]. Even with enough memory it will take much time to extract all eigenvalues. Therefore, a better method called Fast Principal Component Analysis (FPCA), which will calculate in a much faster way, yet brings the same results as PCA does [4].

Besides PCA, many researches also proposed new algorithms to improve recognition rate [5]. Yet, not like other researches that focus on improving algorithm in order to bring a better recognition rate, we are more interested in pre-processing of images, since it may also bring some positive effects on the final performance but in a more simple way. Although many researches also include pre-processing in their study, like adjusting face position, converting into grey-level images, or make some image corrections[6], most of them don't consider a way that enhancing features of a person.

Noticing this ignored aspect, we try to use Fast Fourier transform (FFT) in pre-processing. By using FFT, we process original images and combine amplitude spectrum of one image with phase spectrum of another image to enhance features before extracting eigenvectors. In this study, we use 15 classes in Yale Face Database A, each class containing 11 images of one person. In pre-processing, as we mentioned before, we apply FFT to combine amplitude spectrum of one image with phase spectrum of another image. PCA is used for feature extractor and Support Vector Machine (SVM) is used for classification.

The content of this work is organized as follows. Section 2 deals with our proposed approaches based on different fixed algorithms. Section 3 shows our experiment results. Finally, a conclusion is also given.

\section{Proposed Approaches}

Our method includes three steps. In the first step, we apply FFT for our training data to do pre-processing. The major task in this step is combining amplitude spectrum of one image with phase spectrum of another image into a mixed image. The next step is extracting feature by using FPCA. Finally, SVM is utilized for classification. Fig.1 shows the overall procedure of our work.

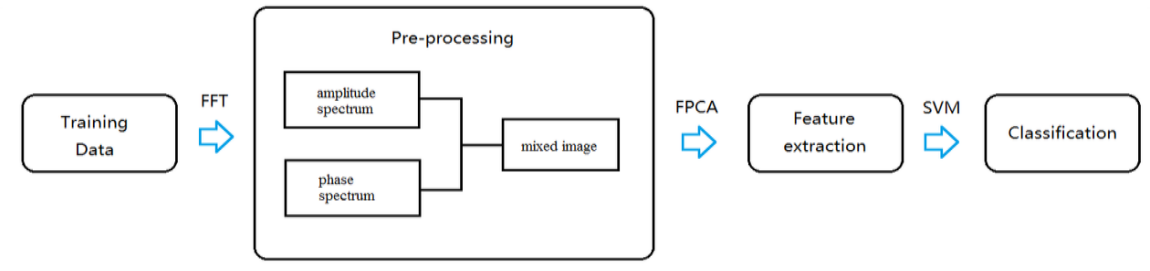

Fig. 1. Overall procedure of face recognition 


\subsection{Pre-processing}

\subsubsection{Fourier Transform}

Virtually everything in the world can be described via a waveform. The Fourier Transform gives us a unique and powerful way of viewing these waveforms.

If we assume the period is $T$, Fourier series should be like:

$$
f(x)=\frac{a_{0}}{2}+\sum_{k=1}^{+\infty}\left(a_{k} \cos n \omega_{0} x+b_{k} \sin n \omega_{0} x\right)
$$

where $\omega_{0}=\frac{2 \pi}{T}=2 \pi u$, and $u=1 / T$, which is the frequency of $f(x)$.

It has already been proven that the first $\mathrm{N}$ terms of Fourier series is the best approximation of anti-derivative $f(t)$ in given energy:

$$
\lim _{N \rightarrow \infty} \int_{\mathbf{0}}^{T}\left|\boldsymbol{f}(\boldsymbol{t})-\left[\frac{a_{0}}{2}+\sum_{k=1}^{+\infty}\left(a_{k} \cos n \omega_{0} x+b_{k} \sin n \omega_{0} x\right)\right]\right|^{2} \boldsymbol{d} \boldsymbol{x}=0
$$

In this paper, because we are dealing with images, so it is obvious for us to know two-dimension Discrete Fourier Transform (DFT):

$$
F(u, v)=\sum_{X=0}^{M-1} \sum_{X=0}^{N-1} f(x, y) e^{-i 2 \pi\left(\frac{u x}{M}+\frac{v y}{N}\right)}
$$

where $u$ and $v$ are variables in domain.

The reason why we need Fast Fourier Transform (FFT) is that for a sequence of $\mathrm{N}$, its definition of DFT transform and inverse transformation is:

$$
\left\{\begin{array}{c}
F(u)=\sum_{X=0}^{N-1} f(x) W_{N}^{u x}, u=0,1, \ldots, N-1, W_{N}=e^{-j \frac{2 \pi}{N}} \\
f(x)=\frac{1}{N} \sum_{u=0}^{N-1} F(u) W_{N}^{-u x}, x=0,1, \ldots, N-1
\end{array}\right.
$$

As we can see in equation (4), it is not hard to find that in order to calculate a sequence of $\mathrm{N}$, it should do $\mathrm{N}^{2}$ plural multiplications and $\mathrm{N}(\mathrm{N}-1)$ plural additions. Yet, the fundament of DFT is not so complex, $\mathrm{W}_{\mathrm{N}}$ has its own periodicity, actually it only has $\mathrm{N}$ values. And also, these $\mathrm{N}$ values have symmetric relation in some degree. Because of periodicity and symmetry of $\mathrm{W}_{\mathrm{N}}$, we could make a conclusion as follows:

$$
\begin{gathered}
W_{N}^{0}=1, W_{N}^{\frac{N}{2}}=1 \\
W_{N}^{N+r}=W_{N}^{r}, W_{N}^{\frac{N}{2}+r}=-W_{N}^{r}
\end{gathered}
$$

where equation (5) represents some special values in matrix $\mathrm{W}$, while equation (6) explains periodicity and symmetry of matrix $\mathrm{W}$.

By utilizing periodicity of matrix $\mathrm{W}$, some terms in DFT calculation can be combined; by utilizing its symmetry, we just need calculate half of W. Based on these two advantages, we could reduce a lot of operations, which is the basic idea of FFT.

\subsubsection{Amplitude Spectrum and Phase Spectrum}

In this paper, we apply FFT to combine amplitude spectrum of one image with phase spectrum of another image to enhance features of images.

The definition of amplitude spectrum:

$$
|F(u, v)|=\left[\operatorname{Re}(u, v)^{2}+\operatorname{Im}(u, v)^{2}\right]^{1 / 2}
$$


Every amplitude spectrum $|F(u, v)|$ of point $(u, v)$ in frequency domain could represent the ratio of sine (or cosine) plane wave to superimposition. Thus amplitude spectrum could reflect the frequency information and have a high practical value in filtering.

The definition of phase spectrum:

$$
\varphi(u, v)=\arg \tan \frac{\operatorname{Im}(u, v)}{\operatorname{Re}(u, v)}
$$

Though it is not so clear to figure out the information about phase spectrum as amplitude spectrum, it contains a kind of ratio relation between real part and imaginary part.

And in addition, we could restore $F(u, v)$ by amplitude spectrum and phase spectrum:

$$
F(u, v)=|F(u, v)| \mathrm{e}^{j \varphi(u, v)}
$$

\subsubsection{Images Combination}

By combining amplitude spectrum of one image with phase spectrum of another image (both images belong to the same class), we got a mixed image that had main information of one image (the one with amplitude spectrum) and minor information of another image (the one with phase spectrum). For improving the recognition rate of face recognition, extracting eigenvectors from a mixed image would be better than extracting eigenvectors from an original image (as there were more features in the that image), therefore enhances the accuracy of SVM's results.

\subsection{Extracting Feature}

PCA is one of the oldest in analysing multiple variables, which is derived from Karhumen-Loeve (KL) transformation. It is Pearson who first proposed PCA in 1901, and in 1963 Karhumen-Loeve made a huge improvement about the original version of PCA [7].

If the image elements are considered to be random variables, then the image may be seen as a sample of a stochastic process. The PCA basis vectors are defined as the eigenvectors of covariance matrix $\mathrm{S}$ :

$$
S=\mathrm{E}\left[X X^{T}\right]
$$

Since the eigenvectors associated with the largest eigenvalues have face-like images, they also are referred to as eigenfaces. If we suppose the eigenvectors of $\mathrm{S}$ are $V_{1}, V_{2}, \ldots, V_{n}$ and are associated respectively with the eigenvalues $m_{1} \geq m_{2} \geq$ $\cdots \geq m_{n}$. Then:

$$
X=\sum_{i=1}^{n} \widehat{x_{i}} v_{i}
$$

However, if the number of dimension is very big, calculating matrix $\mathrm{S}$ will be a tough task that may even make computer gets stuck [8]. Fortunately, there is a good idea to fix it.

Assume that $\mathrm{n}$ is the number of samples, $\mathrm{d}$ is the number of dimension, and $Z_{n \times d}$ is the matrix that every sample in sample matrix $\mathrm{X}$ minus mean Eigenvalue $\vec{m}$, thus scatter matrix $\mathrm{S}$ should be $\left(Z Z^{T}\right)_{d \times d}$. Now consider the matrix 
$R=\left(Z Z^{T}\right)_{n \times n}$, for most occation $\mathrm{d}$ is much greater than $\mathrm{n}$, therefore the size of matrix $\mathrm{R}$ is much smaller than that of matrix $\mathrm{S}$. So we have:

$$
\left(Z Z^{T}\right) \vec{v}=\lambda \vec{v}
$$

And from eq(12) we can get eq(13) by premultiplying $Z^{T}$ :

$$
\left(Z^{T} Z\right)\left(Z^{T} \vec{v}\right)=\lambda\left(Z^{T} \vec{v}\right)
$$

Equation (13) shows that $Z^{T} \vec{v}$ is the eigenvalue of scatter matrix $\mathrm{S}$, which means that we could calculate the smaller matrix R then calculate $Z^{T} \vec{v}$ instead of calculating $Z^{T} \vec{v}$ directly.

To harness PCA in our face recognition system, firstly our program read a sample of training database (m pixel $* \mathrm{n}$ pixel) and saved it in a sample matrix Face Container. Then by applying PCA, we converted $\mathrm{m}^{*} \mathrm{n}$ dimensions matrix into $k$ dimensions matrix, and those $k$ dimensions would represent all the dimensions in a training matrix, which would reduce a lot of operations in the SVM part.

\subsection{Classification}

In this paper, our classification builds on SVM. Unlike artificial neural network, by applying SVM, we could calculate the optimum value based on small samples [9]. The normal maximizing objective function of SVM is like:

$$
L(\alpha)=\sum_{i=1}^{N} \alpha_{i}-\frac{1}{2} \sum_{i=1}^{N} \sum_{j=1}^{N} \alpha_{i} \alpha_{j} y_{i} y_{j} K\left(x_{i}, x_{j}\right)
$$

where $\alpha_{i}$ is Lagrange multipliers.

In order to make a nonlinear projection, a kernel should be chosen [10]. In this study, we choose the radial basis kernel function as follow:

$$
K(x, y)=\exp \left(\left(-\gamma\|x-y\|^{2}\right)\right.
$$

In our method, we should determine both the error cost coefficient $\mathrm{C}$ and $\gamma$ in radial basis kernel function. However, it is very hard using $\mathrm{C}$ and $\gamma$ to make an expression by normal optimizing strategy. Fortunately, Doctor Chih-Jen Lin provides a very good way to solve this problem.

The main task in classification by using SVM is to determine $\mathrm{C}$ and $\gamma$, as normal optimizing strategies usually doesn't work well. Yet, in this aspect, Doctor Chih-Jen Lin proposed a program called grid.py to fix it. By using this program, we finally determine that the optimum value of $\mathrm{C}$ is 128 , and the optimum value of $\gamma$ is 0.0078125 .

\section{Experimental Results}

In this section, we will evaluate the performance of proposed approaches for face recognition. We first did FFT for the entire training database, extracted PCA feature vectors and then applied each feature extraction method with SVM. All the works were done in Matlab2011b in a PC with $2.5 \mathrm{GHz}$ Intel Core i5 and 4GB 1600MHz DDR3 RAM. 


\subsection{Face Database}

In this study, our training data is from Yale face database. The Yale database contains 165 images of 15 individuals. Each individual has 11 images with different facial expressions or configurations: normal, happy, sad, sleepy, surprised, and wink; centre-light, left-light, and right-light; with and without glasses.

\subsection{Experiments on Yale Database and discussion}

In the experiment, we firstly used FFT to combine amplitude spectrum of one image with phase spectrum of the next image (the last one will combine with the first one). All the mixed images we got are shown in Fig.2 compared with original images.

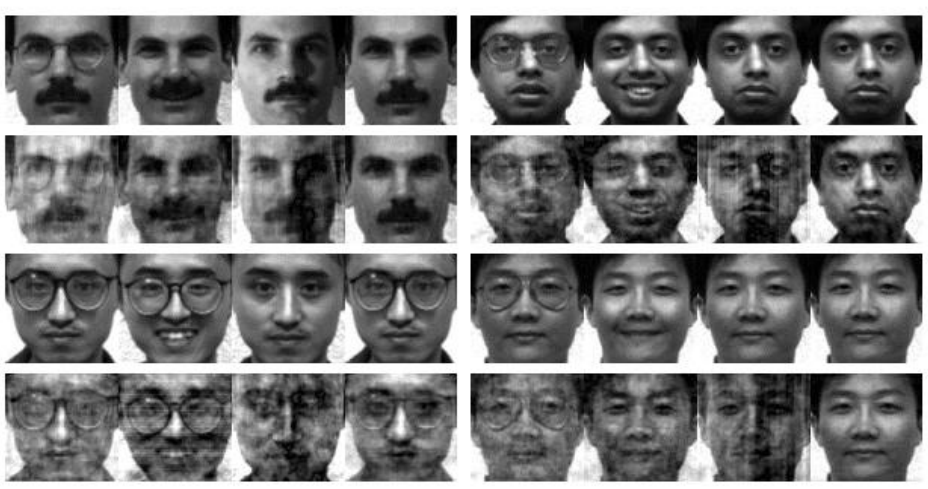

Fig. 2. Original images (above) and after FFT processing (below)

To evaluate our method, we compare and contrast the results of PCA+SVM and that of FFT+PCA+SVM. And k (dimensions of Eigenvector)is set to 20, 30, 40 respectively. The results are shown in Table 1.

Table 1. Recognition rate comparison of different approaches on Yale database

\begin{tabular}{ccccc}
$\begin{array}{c}\text { Training } \\
\text { samples } \\
\text { per } \\
\text { individual }\end{array}$ & $\begin{array}{c}\text { Value } \\
\text { of k }\end{array}$ & $\begin{array}{c}\text { PCA+SVM } \\
\text { Recognition rate } \\
(\%)\end{array}$ & $\begin{array}{c}\text { FFT+PCA+SVM } \\
\text { Recognition rate } \\
(\%)\end{array}$ & $\begin{array}{c}\text { Improvement } \\
\text { rate (\%) }\end{array}$ \\
\hline \multirow{3}{*}{3} & 20 & 81.905 & 83.81 & 2.326 \\
& 30 & 78.095 & 80 & 2.439 \\
& 40 & 81.905 & 83.81 & 2.326 \\
4 & 20 & 84.127 & 85.714 & 1.886 \\
& 30 & 84.127 & 85.714 & 1.886 \\
& 40 & 82.540 & 84.127 & 1.923 \\
5 & 20 & 84.211 & 90.79 & 7.813 \\
& 30 & 85.526 & 89.474 & 4.616 \\
& 40 & 84.211 & 93.421 & 10.937
\end{tabular}


As we can see from Table 1, the results produced by FFT $+\mathrm{PCA}+\mathrm{SVM}$ are better than that by FFT+PCA+SVM. And we can learn that when training samples of one individual is 5, the peak recognition rate is $93.421 \%$. And in one reference [11], the peak recognition rate is 86.67 , whose experiment was also based on Yale database and PCA and used 6 samples for training.

We also compare our results with DCV and KDDA, as shown in Fig 3

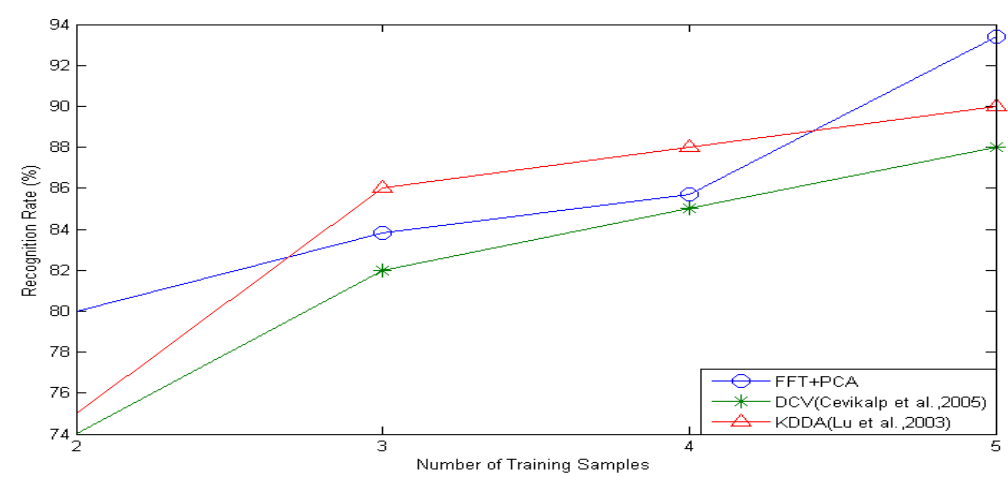

Fig. 3. Experiment comparison on Yale database

From Fig.3, we learn that when the number of training samples is small, recognition rates of three approaches are close. However, when the number of training becomes greater, our method is better than DCV and KDDA.

Since we have an original Yale database and FFT-YALE database, we did another experiment that we chose FFT-Yale database as training database and chose original Yale database as testing database. The results are shown in Table 2.

Table 2. Recognition rate based on FFT-Yale database as training database and original Yale database as testing database

Value of $\mathrm{k}$

Recognition rate $(\%)$

$\begin{array}{cc}20 & 96.364 \\ 30 & 98.7879 \\ 40 & 97.576 \\ 50 & 98.7879 \\ 100 & 98.1818\end{array}$

From Table 2, we can see that recognition rate is very high. And it indicates that our proposed approach is encouraging.

\section{Conclusions}

We present a full system of face recognition (FFT+PCA+SVM). Noticing that few researches focus on pre-processing of images, which will also improve the performance of classification, we apply Fourier Transform as a way to do 
pre-processing. Via FFT, we combine amplitude spectrum of one image with phase spectrum of another image as a mixed image. Then we harness PCA to extract Eigenvectors and use SVM as a classifier.

Our experiment is based on Yale database, and we compare our approach with others. The results indicate that our approach is effective.

\section{Acknowledgement}

This work is supported by the National Natural Science Foundation of China (Grant No. 61263043) and the Department of Education of Yunnan Province (Grant No. 2011Z020) and the Key Discipline Foundation of School of Software of Yunnan University (Grant No.2012SE103).

\section{Reference}

1. Eftekhari A, Forouzanfar M, Moghaddam HA, Alirezaie J, Block-wise 2D kenel PCA/LDA for face recognition, Information Processing Letters 110 (2010), 761-766

2.Cevikalp H, Wilkes M, Barkana A, Discriminative common vectors for face recognition, IEEE Transactions on Pattern Analysis and Machine Intelligence 27 (1) (2005) 4-13

3. Cao LJ, Chua KS, Chong WK, et al., A comparison of PCA, KPCA, and ICA for dimensionality reduction in support vector machine, Neurocomputing 55 (2003) 321-336.

4. Wang $\mathrm{H}, \mathrm{Li}$ SZ, Wang Y, Face recognition under varying lighting conditions using self-quotient image, in: Proceedings of the IEEE International Conference on Automatic Face and Gesture Recognition, 2004, pp. 819-824.

5. Adini Y, Moses Y, Ullman S, Face recognition: the problem of compensating for changes illumination direction, IEEE Transaction on Pattern Analysis and Machine Intelligence 19 (1997) 721-732.

6. Levada LMA, Correa DC, Salvadeo DHP, Saito JH, Nelson DA, Novel approaches for face recognition: template-matching using dynamic time warping and LSTM Neural Network Supervised Classification, in: International Conference on Systems, Signals and Image Processing, 2008, pp. 241-244.

7. Cao X, Shen W, Yu LG, Wang YL, Yang JY, Zhang ZW, Illumination invariant extraction for face recognition using neighboring wavelet coefficients, Pattern Recognition 45(2012), 1299-1305

8. Liu C, Wechsler H, Gabor feature based classification using the enhanced fisher linear discriminant model for face recognition, IEEE Transactions on Image Processing 11 (2002) 467-476

9. Wen Y, He LH, Shi PF, Face recognition using difference vector plus KPCA, Digital Signal Processing 22 (2012), 140-146

10. Taylor JS, Cristianini N, Kernel Methods for Pattern Analysis, Cambridge University Press, 2004.

11. Wang HY, Leng Y, Wang ZF, Wu XJ, Application of image correction and bit-plane fusion in generalized PCA based face recognition. Pattern Recognition Letters 28(2007) 2352-2358 